\title{
The Space Structure, Force Fields, and Dark Matter
}

\author{
Ding-Yu Chung ${ }^{1}$, Volodymyr Krasnoholovets ${ }^{2}$ \\ ${ }^{1}$ Utica, Michigan, USA \\ ${ }^{2}$ Institute of Physics, National Academy of Sciences, Kyiv, Ukraine \\ Email: dy_chung@yahoo.com, krasnoh@iop.kiev.ua
}

Received January 28, 2013; revised March 1, 2013; accepted March 11, 2013

Copyright (c) 2013 Ding-Yu Chung, Volodymyr Krasnoholovets. This is an open access article distributed under the Creative Commons Attribution License, which permits unrestricted use, distribution, and reproduction in any medium, provided the original work is properly cited.

\begin{abstract}
It is proposed that the digital space structure consists of attachment space (denoted as 1) for rest mass and detachment space (denoted as 0 ) for kinetic energy. Attachment space attaches to object permanently with zero speed, and detachment space detaches from the object at the speed of light. The combination of attachment space and detachment space brings about the three structures: binary lattice space, miscible space, and binary partition space. Binary lattice space, (1 $0)_{n}$, consists of repetitive units of alternative attachment space and detachment space. In miscible space, attachment space is miscible to detachment space without separation. Binary partition space, $(1)_{n}(0)_{n}$, consists of separated continuous phases of attachment space and detachment space. Binary lattice space, miscible space, and binary partition space constitute quantum mechanics, special relativity, and the extreme force fields, respectively. Through the detachment space, a higher dimensional particle in attachment space is sliced into infinitely surrounding a lower dimensional core attachment space, resulting in a particle surrounding by gauge field in the form of binary lattice space. The 10d particle can be sliced into 9d, 8d, 7d, 6d, 5d, and 4d particles equally by mass, corresponding to baryonic particle as $4 d$ and dark matter as other 5 particles, so the ratio between baryonic matter and dark matter is 1 to 5 , in agreement with the observed ratio. At extreme conditions, such as extremely low temperature, the gauge force field in the form of binary lattice space is transformed into the extreme force field in the form of binary partition space to explain extreme phenomena, such as superconductivity.
\end{abstract}

Keywords: Space Structure; Quantum Mechanics; Force Fields; Dark Matter; Superconductivity

\section{Introduction}

The conventional explanation of the hidden extra space dimensions is the compactization of the extra space dimensions. For example, six space dimensions become hidden by the compactization, so space-time appears to be four dimensional. Bounias and Krasnoholovets [1] propose another explanation of the reduction of $>4 \mathrm{D}$ space-time into $4 \mathrm{D}$ space-time by slicing $>4 \mathrm{D}$ spacetime into infinitely many $4 \mathrm{D}$ slices surrounding the $4 \mathrm{D}$ core particle. Such slicing of $>4 \mathrm{D}$ space-time is like slicing 3D object into 2D object in the way stated by Michel Bounias as follows: "You cannot put a pot into a sheet without changing the shape of the $2 \mathrm{D}$ sheet into a $3 \mathrm{D}$ dimensional packet. Only a 2D slice of the pot could be a part of sheet”.

One way to describe the slicing of space dimension is to have the space structure consisting of attachment space and detachment space. Attachment space is the space attaching to an object, such as $3 \mathrm{D}$ attachment space attaching to a pot described above. Detachment space is the space cutting attachment space into numerous attachment space slices, such as detachment space cutting 3D attachment space into numerous 2D attachment space slices. Such 2D attachment space slices with attached objects, therefore, are separated by 2D detachment space gaps without attached objects.

In this paper, the space structure consists of attachment space and detachment space. This space structure explains the difference between quantum mechanics and special relativity, the different force fields, the difference between baryonic matter and dark matter, and the ratio between baryonic matter and dark matter.

\section{The Space Structure}

The digital space structure [2-4] consists of attachment space (denoted as 1 ) and detachment space (denoted as 0 ). Attachment space attaches to object permanently with zero speed. Detachment space detaches from the object at 
the speed of light. Attachment space relates to rest mass, while detachment space relates to kinetic energy. Different stages of our universe have different space structures. The transformation between mass (massive particle) in attachment space and kinetic energy (massless particle) in detachment space is through the scalar Goldstone boson. For example, massive particles with $\mathrm{n}$ units of attachment space, denoted as $(1)_{n}$, are converted into massless particles with $\mathrm{n}$ units of detachment space, denoted as $(0)_{n}$ through the Goldstone bosons. The addition of the Goldstone bosons to massless particles in detachment space results in massive particles in attachment space, while the removal of removal of the Goldstone bosons in attachment space leads to massless particles in detachment space.

$$
\begin{aligned}
& \text { massive particles in }(1)_{n} \\
& \stackrel{+ \text { Goldstone boson }}{\longleftarrow} \text { massless particles in }(0)_{n} \\
& \text { massive particles in }(1)_{n} \\
& \stackrel{- \text { Goldstone boson }}{\longrightarrow} \text { massless particles in }(0)_{n}
\end{aligned}
$$

The Goldstone boson provides the longitudinal degree of freedom for massive particle in attachment space. The Goldstone boson itself is a zero-energy virtual particle by taking energy from and returning energy to the object transformed by the Goldstone boson. The Goldstone boson in the Standard Model for electroweak interaction is the Higgs boson.

The combination of attachment space (1) and detachment space (0) brings about three different space structures: miscible space, binary partition space, and binary lattice space for four-dimensional space-time as below.

$$
\begin{aligned}
& \begin{array}{l}
(1)_{n}+(0)_{n} \underset{\text { combination }}{\longrightarrow} \\
\text { attachment space }
\end{array} \\
& (10)_{n} \text { binary lattice space, } \\
& (1+0)_{n} \text { miscible space, or } \\
& (1)_{n}(0)_{n} \text { binary partition space }
\end{aligned}
$$

Binary lattice space, $\left(\begin{array}{ll}1 & 0\end{array}\right)_{n}$, consists of repetitive units of alternative attachment space and detachment space. Thus, binary lattice space consists of multiple quantized units of attachment space separated from one another by detachment space. In miscible space, attachment space is miscible to detachment space, and there is no separation of attachment space and detachment space. Binary partition space, $(1)_{n}(0)_{n}$, consists of separated continuous phases of attachment space and detachment space.

Binary lattice space consists of multiple quantized units of attachment space separated from one another by detachment space. An object exists in multiple quantum states separated from one another by detachment space. Binary lattice space is the space for wavefunction. In wavefunction,

$$
|\Psi\rangle=\sum_{i=1}^{n} c_{i}\left|\phi_{i}\right\rangle
$$

Each individual basis element, $\left|\phi_{i}\right\rangle$, attaches to attachment space, and separates from the adjacent basis element by detachment space. Detachment space detaches from object. Binary lattice space with $n$ units of four-dimensional, ( $\left.\begin{array}{ll}0 & 1\end{array}\right)_{n}$, contains $n$ units of basis elements.

Neither attachment space nor detachment space is zero in binary lattice space. The measurement in the uncertainty principle in quantum mechanics is essentially the measurement of attachment space and momentum in binary lattice space: large momentum has small non-zero attachment space, while large attachment space has low non-zero momentum. In binary lattice space, an entity is both in constant motions as wave for detachment space and in stationary state as a particle for attachment space, resulting in the wave-particle duality.

Detachment space contains no object that carries information. Without information, detachment space is outside of the realm of causality. Without causality, distance (space) and time do not matter to detachment space, resulting in non-localizable and non-countable spaceime. The requirement for the system (binary lattice space) containing non-localizable and non-countable detachment space is the absence of net information by any change in the space-time of detachment space. All changes have to be coordinated to result in zero net information. This coordinated non-localized binary lattice space corresponds to nilpotent space. All changes in energy, momentum, mass, time, space have to result in zero as defined by the generalized nilpotent Dirac equation by B. M. Diaz and P. Rowlands [5].

$$
\begin{aligned}
& (\mp k \partial / \partial t \pm i \nabla+j m)( \pm i k E \pm i p+j m) \\
& \cdot \exp i(-E t+p \cdot r)=0
\end{aligned}
$$

where $E, p, m, t$ and $r$ are respectively energy, momentum, mass, time, space and the symbols $\pm 1, \pm i, \pm i, \pm j, \pm k$, $\pm i, \pm j, \pm \mathrm{k}$, are used to represent the respective units required by the scalar, pseudoscalar, quaternion and multivariate vector groups. The changes involve the sequential iterative path from nothing (nilpotent) through conjugation, complexification, and dimensionalization. The nonlocal property of binary lattice space for wavefunction provides the violation of Bell inequalities [6] in quantum mechanics in terms of faster-than-light influence and indefinite property before measurement. The non-locality in Bell inequalities does not result in net new information.

In binary lattice space, for every detachment space, there is its corresponding adjacent attachment space. Thus, no part of the object can be irreversibly separated 
from binary lattice space, and no part of a different object can be incorporated in binary lattice space. Binary lattice space represents coherence as wavefunction. Binary lattice space is for coherent system. Any destruction of the coherence by the addition of a different object to the object causes the collapse of binary lattice space into miscible space. The collapse is a phase transition from binary lattice space to miscible space.

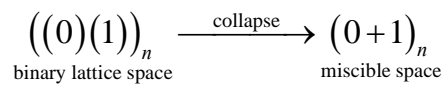

Another way to convert binary lattice space into miscible space is gravity. Penrose [7] pointed out that the gravity of a small object is not strong enough to pull different states into one location. On the other hand, the gravity of large object pulls different quantum states into one location to become miscible space. Therefore, a small object without outside interference is always in binary lattice space, while a large object is never in binary lattice space.

The information in miscible space is contributed by the combination of both attachment space and detachment space, so information can no longer be non-localize. Any value in miscible space is definite. All observations in terms of measurements bring about the collapse of wavefunction, resulting in miscible space that leads to eigenvalue as definite quantized value. Such collapse corresponds to the appearance of eigenvalue, $E$, by a measurement operator, $H$, on a wavefunction, $\Psi$.

$$
H \Psi=E \Psi
$$

In miscible space, attachment space is miscible to detachment space, and there is no separation of attachment space and detachment space. In miscible space, attachment space contributes zero speed, while detachment space contributes the speed of light. A massless particle, such as photon, is on detachment space continuously, and detaches from its own space continuously. For a moving massive particle consisting of a rest massive part and a massless part, the massive part with rest mass, $m_{0}$, is in attachment space, and the massless part with kinetic energy, $K$, is in detachment space. The combination of the massive part in attachment space and massless part in detachment leads to the propagation speed in between zero and the speed of light.

To maintain the speed of light constant for a moving particle, the time $(t)$ in moving particle has to be dilated, and the length $(L)$ has to be contracted relative to the rest frame.

$$
\begin{aligned}
& t=t_{0} / \sqrt{1-v^{2} / c^{2}}=t_{0} \gamma, \\
& L=L_{0} / \gamma, \\
& E=K+m_{0} c^{2}=\gamma m_{0} c^{2}
\end{aligned}
$$

where $\gamma=1 / \sqrt{1-v^{2} / c^{2}}$ is the Lorentz factor for time dilation and length contraction, $E$ is the total energy, and $K$ is the kinetic energy.

Binary partition space, $(1)_{n}(0)_{n}$, consists of separated continuous phases of attachment space and detachment space. It is for extreme force fields under extreme conditions such as near the absolute zero temperature. It will be discussed in the Section 3 to explain extreme phenomena such as superconductivity.

\section{Baryonic Matter, Dark Matter, and Gauge Force Fields}

As described in Reference [2], at the beginning of the current universe, the 10d particle universe was sliced into six particles: 9d, 8d, 7d, 6d, 5d, and 4d equally by mass. Baryonic matter is $4 \mathrm{~d}$, while dark matter consists of the other five types of particles (9d, 8d, 7d, 6d, and 5d). The mass ratio of dark matter to baryonic matter is 5 to 1 in agreement with the observation [8] showing the universe consists of $22.7 \%$ dark matter, $4.56 \%$ baryonic matter, and $72.8 \%$ dark energy.

Detachment space (0) involves in the slicing of dimensions. Attachment space is denoted as 1. For example, the slicing of $10 \mathrm{~d}$ particles into $4 \mathrm{~d}$ particles is as follows.

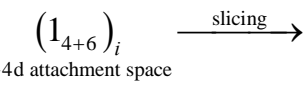

$$
\begin{aligned}
& \underset{4 d \text { core attachment space }}{\left(1_{4}\right)_{1}}+\sum_{6 \text { types of } 4 \mathrm{~d} \text { units }}^{6}\left(\left(0_{4}\right)\left(1_{4}\right)\right)_{j, 6}
\end{aligned}
$$

The two products of the slicing are the 4d-core attachment space and 6 types of $4 d$ quantized units. The $4 d$ core attachment space surrounded by 6 types of many $(j)$ 4d-quantized units corresponds to the core particle surrounded by 6 types of many small $4 \mathrm{~d}$ particles.

Therefore, the transformation from $\mathrm{d}$ to $d-n$ involves the slicing of a particle with d dimension into two parts: the core particle with $d-n$ dimension and the $n$ dimensions that are separable from the core particle. Such $n$ dimensions are denoted as $n$ "dimensional orbitals", which become gauge force fields [9]. The sum of the number of dimensions for a particle and the number of dimensional orbitals (DO's) is equal to 11 (including gravity) for all particles with dimensions. Therefore,

$$
F_{d}=F_{d-n}+(11-d+n) \text { DO's }
$$

where $11-d+n$ is the number of dimensional orbitals (DO's) for $F_{d-n}$. Thus, 10d particles can transformed into $9 d, 8 d, 7 d, 6 d, 5 d$, and $4 d$ core particles, which have 2, 3, 4, 5, 6, and 7 separable dimensional orbitals, respectively. Baryonic matter particle $4 \mathrm{~d}$ has gravity and six other dimensional orbitals as gauge force fields

The dimensional orbitals of baryonic matter provide the base for the periodic table of elementary particles to calculate accurately the masses of all elementary parti- 
cles, including quarks, leptons, and gauge bosons [9].

The lowest dimensional orbital is for electromagnetism. Baryonic matter is the only one with the lowest dimensional orbital for electromagnetism. With higher dimensional orbitals, dark matter does not have this lowest dimensional orbital. Without electromagnetism, dark matter cannot emit light, and is incompatible to baryonic matter, like the incompatibility between oil and water. The incompatibility can be explained by the repulsive part of the gravity from an elastic interaction between the two matters $[10,11]$. The incompatibility between dark matter and baryonic matter leads to the inhomogeneity (like emulsion), resulting in the formation of galaxies, clusters, and superclusters [11]. Dark matter has not been found by direct detection because of the incompatibility that does not allow the direct contact with dark matter by baryonic matter.

\section{Extreme Force Field}

Under extreme conditions such as the absolute zero temperature, binary lattice space for a gauge force field undergoes a phase transition to become binary partition space for the extreme force fields $[4,11]$.

At zero temperature, binary lattice space for a gauge force field undergoes a quantum space phase transition to become binary partition space. In binary partition space, detachment space and attachment space are in two separate continuous regions as follows.

$$
\begin{gathered}
\underset{\substack{\left(1_{4}\right)_{m} \\
\text { particle }}}{\left(1_{4}\right)_{m}}+\sum_{\substack{k=1 \\
\text { boson field in binary lattice space }}}^{k}\left(\left(0_{4}\right)\left(1_{4}\right)\right)_{n, k} \rightarrow \\
\text { extreme particle } \\
\text { extreme boson field in binary lattice space }
\end{gathered}
$$

The force field in binary lattice space is gauge boson force field, the force field in binary partition space is denoted as "extreme boson force field". The detachment space in extreme boson field is the vacuum core, while extreme bosons attached to attachment space form the extreme boson shell. Gauge boson force field has no boundary, while the attachment space in the binary partition space acts as the boundary for extreme boson force field. Extreme boson field is like a bubble with core vacuum surrounded by membrane where extreme bosons locate.

The overlapping (connection) of two extreme bosons from two different sites results in "extreme bond". The product is "extreme molecule". An example of extreme molecule is Cooper pair, consisting of two electrons linked by extreme bond. Another example is superfluid, consisting of molecules linked by extreme bonds. Extreme bonds can be also formed among the sites in a lattice, resulting in extreme lattice. Extreme lattice is su- perconductor. Extreme boson force is incompatible to gauge boson force field. The incompatibility of extreme boson force field and gauge boson force field manifests in the Meissner effect, where superconductor (extreme lattice) repels external magnetism. The energy (stiffness) of extreme boson force field can be determined by the penetration of boson force field into extreme boson force field as expressed by the London equation for the Meissner effect.

$$
\nabla^{2} H=-\lambda^{-2} H,
$$

where $H$ is an external boson field and $\lambda$ is the depth of the penetration of magnetism into extreme boson shell.

\section{Summary}

It is proposed that the digital space structure consists of attachment space (denoted as 1) for rest mass and detachment space (denoted as 0) for kinetic energy. Attachment space attaches to object permanently with zero speed, and detachment space detaches from the object at the speed of light. The combination of attachment space and detachment space brings about the three structures: binary lattice space, miscible space, and binary partition space. Binary lattice space, $\left(\begin{array}{ll}1 & 0\end{array}\right)_{n}$, consists of repetitive units of alternative attachment space and detachment space. In miscible space, attachment space is miscible to detachment space without separation. Binary partition space, $(1)_{n}(0)_{n}$, consists of separated continuous phases of attachment space and detachment space. Binary lattice space, miscible space, and binary partition space constitute quantum mechanics, special relativity, and the extreme force fields, respectively. Through the detachment space, a higher dimensional particle in attachment space is sliced into infinitely surrounding a lower dimensional core attachment space, resulting in a particle surrounding by gauge field in the form of binary lattice space. The $10 \mathrm{~d}$ particle can be sliced into 9d, 8d, 7d, 6d, 5d, and 4d particles equally by mass, corresponding to baryonic particle as $4 \mathrm{~d}$ and dark matter as other 5 particles, so the ratio between baryonic matter and dark matter is 1 to 5 , in agreement with the observed ratio. At extreme conditions, such as extremely low temperature, the gauge force field in the form of binary lattice space is transformed into the extreme force field in the form of binary partition space to explain extreme phenomena, such as superconductivity.

\section{REFERENCES}

[1] M. Bounias and V. Krasnoholovets, "Scanning the Structure of Ill-Known Spaces: Part 1. Founding Principles about Mathematical Constitution of Space,” The International Journal of Systems and Cybernetics, Vol. 32, No. 7/8, 2003. pp. 945-975. doi: $10.1108 / 03684920310483126$ 
[2] D. Chung and V. Krasnoholovets, "The Cosmic Organism Theory,” Scientific Inquiry, Vol. 8, 2007, pp. 165-182. arXiv: physics/0512026

[3] V. Krasnoholovets and D. Y. Chung, "The Space Structure, Force Fields and Quantum Mechanics," International Journal of Anticipatory Computing Systems, Vol. 839, 2006, pp. 191-197.

http://inerton.org/Inerton_Theory___Papers_-_Sub-micros copic_Mechanics_files/30_Chung\&Krasn_1.pdf

[4] D. Chung and V. Krasnoholovets, "The Quantum Space Phase Transitions for Particles and Force Fields," Progress in Physics, Vol. 4, 2006, pp. 74-77. http://www.ptep-online.com/index_files/2006/PP-06-14.P DF

[5] B. M. Diaz and P. Rowlands, "A Computational Path to the Nilpotent Dirac Equation,” American Institute of Physics Proceedings of the International Conference of Computing Anticipatory Systems, 2003, pp. 203-218. arXiv:cs/0209026

[6] J. S. Bell, "On the Einstein-Podolsky-Rosen Paradox," Physics, Vol. 1, 1964, pp. 195-199.
[7] R. Penrose, "Wavefunction Collapse as a Real Gravitational Effect,” In: A. Fokas, A. Grigoryan, T. Kibble and B. Zegarlinski, Eds., Mathematical Physics, Imperial College, London, 2000, pp. 266-282.

[8] N. Jarosik, et al., "Seven-Year Wilson Microwave Anisotropy Probe (WMAP) Observations: Sky Maps, Systematic Errors, and Basic Results,” 2010.

http://lambda.gsfc.nasa.gov/product/map/dr4/pub_papers/ sevenyear/basic_results/wmap_7yr_basic_results.pdf

[9] D. Chung, "The Periodic System of Elementary Particles and the Composition of Hadrons," Speculations in Science and Technology, Vol. 20, 1997, pp. 259-268. http://arxiv.org/ftp/hep-th/papers/0111/0111147.pdf

[10] V. Krasnoholovets, "Dark Matter as Seen from the Physical Point of View,” Astrophysics Space Science, Vol. 335, No. 2, 2011, pp. 619-627. doi:10.1007/s10509-011-0774-y

[11] D. Chung, “The Unified Theory of Physics,” 2012. arXiv:hep-th/0201115 